\title{
Correlação da massa e porcentagem de gordura com a idade na Distrofia Muscular de Duchenne
}

\author{
Correlation between mass and percentage of fat according to the age \\ in Duchenne Muscular Dystrophy
}

\author{
Fátima Aparecida Caromano ${ }^{[a]}$, Clarice Tanaka ${ }^{[b]}$, Sílvia Maria Amado João ${ }^{[c]}$, \\ Ana Patrícia Kamisaki ${ }^{[\mathrm{d}]}$, Kelly Cristina Yano ${ }^{[\mathrm{e}]}$, Maiza Ritomy Ide ${ }^{[\mathrm{f}]}$
}

[a] Doutora, Faculdade de Medicina da Universidade de São Paulo (USP), São Paulo, SP - Brasil, e-mail: caromano@usp.br

[b] Graduada em Fisioterapia, com Mestrado e Doutorado pela Universidade de São Paulo (USP), Pós-Doutorado pela McGill University e pela Université de Montréal, professora titular do Departamento de Fisioterapia, Fonoaudiologia e Terapia Ocupacional da Faculdade de Medicina da USP, São Paulo, SP - Brasil, e-mail: cltanaka@uol.com.br

[c] Graduada em Fisioterapia, Mestre e Doutora em Ciências (Biologia Celular e Tecidual) pela Universidade de São Paulo (USP), São Paulo, SP - Brasil, e-mail: smaj@usp.br

[d] Iniciação Científica, Laboratório de Fisioterapia e Comportamento, Faculdade de Medicina da Universidade de São Paulo (USP), São Paulo, SP - Brasil, e-mail: patkamisaki@usp.br

[e] Graduada em Fisioterapia pela Universidade Estadual do Oeste do Paraná (UNIOESTE), Cascavel, PR - Brasil, e-mail: kelly_ yano@yahoo.com.br

[f] Graduada em Fisioterapia pela Universidade Estadual de Londrina (UEL), Mestre em Ciências da Reabilitação e Doutora em Reumatologia pela Faculdade de Medicina da Universidade de São Paulo (USP), Pós-Doutora pela Universidad de Cantabria (Espanha), professora adjunta do Curso de Fisioterapia da Universidade Estadual do Oeste do Paraná (UNIOESTE), Cascavel, PR - Brasil, e-mail: maizaide@hotmail.com

\section{Resumo}

Introdução: A Distrofia Muscular de Duchenne (DMD) é uma desordem genética, caracterizada pela perda progressiva e irreversível da musculatura esquelética. Objetivo: Este estudo objetiva correlacionar a porcentagem e a massa de gordura com a idade em pacientes com DMD. Metodologia: Foram selecionados 68 indivíduos com idades entre 5 e 20 anos, com diagnóstico molecular de certeza para DMD, residentes na cidade de São Paulo. Todos foram submetidos à mensuração do peso e altura e também ao teste de análise de composição corporal com o uso da bioimpedância, no período da manhã, todas no mesmo dia. Resultados e discussão: Os resultados foram analisados agrupando os indivíduos em quartis de idade e mostraram um índice de massa corpóreo (IMC) de $21 \pm 8 \mathrm{~kg} / \mathrm{m}^{2}$. Assim, observou-se que, com a idade e o grau de sedentarismo imposto pela doença, houve um acúmulo de gordura corporal 
e perda de massa magra. Conclusão: São necessários mais estudos relacionados às características nutricionais desses indivíduos, para que se esclareçam melhor os efeitos da doença e da alimentação no ganho de porcentagem e massa de gordura.

Palavras-chave: Distrofia muscular de Duchenne. Composição corporal. Idade.

\begin{abstract}
Introduction: The Duchenne Muscular Dystrophy (DMD) is a genetic disorder, characterized by the progressive and irreversible loss of skeletal muscle. Purpose: This study aims to correlate the percentage and mass of fat to the age of patients with DMD. Methods: It was selected 68 individuals aged between 5 and 20 years, with molecular diagnosis of certainty for DMD, residents in the city of São Paulo. All the them were submitted to weight and height measurement and also to the test of body composition analysis with the use of bioelectrical impedance, in the morning period, all the same day. Results and discussion: The results were analyzed by grouping individuals into quartiles of age and showed a body mass index (BMI) of $21 \pm 8 \mathrm{~kg} / \mathrm{m}^{2}$. Thus, it was observed that with the age and the degree of inactivity imposed by the disease, there was an accumulation of body fat and loss of lean mass. Conclusion: Further studies related to the nutritional characteristics of these individuals are necessary to clarify the effects of disease and food in the gain on the percentage of body and mass fat.
\end{abstract}

Keywords: Duchenne muscular Dystrophy. Body composition. Age.

\title{
Introdução
}

A Distrofia Muscular de Duchenne (DMD) é a segunda desordem genética mais comum em humanos. Constitui um distúrbio genético de caráter recessivo, com alta taxa de mutação, em um gene localizado no cromossomo Xp21, responsável por um defeito na membrana muscular que causa ausência ou diminuição da produção de distrofina, proteína responsável, entre outras funções, pela integridade da membrana basal da fibra muscular. O quadro clínico da DMD caracteriza-se principalmente pela perda progressiva e irreversível da musculatura esquelética, morte celular com a substituição de células musculares por fibrose e gordura $(1,2,3)$.

As manifestações clínicas estão presentes desde o nascimento, mas se tornam evidentes entre os 3 e 5 anos de idade. Caracterizam-se por perda progressiva da força muscular, com predileção pelos músculos proximais dos membros e pelos músculos flexores do pescoço (4). É comum apresentarem comprometimento intelectual e terem degeneração miocárdica, que desencadeia insuficiência cardíaca direita insidiosa (5).

De acordo com Willing (6), a DMD é uma desordem muscular progressiva, cujo curso pode ser afetado por graves problemas nutricionais. A obesidade, que pode afetar a vida cotidiana de meninos préadolescentes, muitas vezes ocorre na mesma época que a perda da deambulação, que pode ocorrer entre 7 e 12 anos de idade (7).

Pacientes com DMD aumentam de peso conforme a doença progride, não sendo incomum os meninos terem maior peso e menor massa muscular que garotos normais da mesma idade (8).

O diagnóstico precoce só é possível com o reconhecimento dos sintomas, solicitação do teste de triagem dosagem sérica de creatinofosfoquinase e encaminhamento para especialistas capazes de realizarem o diagnóstico definitivo, por meio de análise molecular e/ou biopsia muscular com imuno-histoquímica $(9,10)$.

Araújo (11) mostra que há geralmente há demora no diagnóstico de DMD, ocasionada pela pouca importância dada aos sinais precoces, pela triagem nas condutas, ou pela dificuldade de acesso aos locais onde o diagnóstico correto poderia ser oferecido.

A obesidade é comum na DMD (12-16). Um estudo com nove crianças portadoras de DMD com idade de 6 a 12 anos mostrou que cerca da metade desenvolveu obesidade, o que constitui sobrecarga ao trabalho desenvolvido pela musculatura esquelética (17). Segundo Hankard (18), a obesidade pode ser desencadeada 
pelo desequilíbrio entre o consumo e o gasto de energia dessas crianças, e acentuada pelo sedentarismo, a partir do momento que os movimentos da criança se tornam comprometidos.

Bahareh (19) afirma que os portadores de DMD possuem nutrição inadequada por causa da ausência de informação sobre a quantidade que deve ser consumida de caloria (proteína, carboidrato e gordura), vitaminas e minerais, e também pela mobilidade prejudicada pela doença. Estudo realizado pelos autores com 29 pacientes com DMD e 22 pacientes com outras distrofias demonstrou uma correlação entre massa e força muscular e características nutricionais desses indivíduos, associadas principalmente ao desequilíbrio entre a síntese e degradação de proteína, característico da doença.

De acordo com Satomura (20), observa-se perda de peso corporal parodoxical em portadores de DMD a partir dos 13 anos de vida, em função de ativação simpática, que resulta no aumento da produção de calor no tecido adiposo marrom e, por consequência, no aumento do consumo de energia. Esse processo requer a ampliação da ingestão calórica quando se deseja a manutenção do peso corporal, importante na melhora do prognóstico da função motora respiratória.

Um estudo apresentado por Berlit (21), utilizando análise de composição corporal por meio de bioimpedância, com 21 garotos portadores de DMD e 20 normais (grupo controle), encontrou que a massa de gordura corporal era maior no grupo de $\operatorname{DMD}(38,8 \pm 7,1$ contra $16,4 \pm 5,3)$, enquanto que a massa livre de gordura diminuiu em comparação com o controle $(46,9 \pm 11,6$ contra 58,9 $\pm 10,0)$. Esse estudo concluiu haver uma correlação significativa entre gordura corporal e a duração de vida das crianças portadoras de DMD, demonstrando que quanto maior a porcentagem de gordura corporal, menor o tempo de vida. Segundo os autores, porcentagem de gordura corporal pode então ser um preditor de sobrecarga e expectativa de vida.

Estudo realizado por McDonald (22) utilizando o método dual energy x-ray absorptionmetry e a técnica de bioimpedância, com 46 participantes (12 não obesos, 19 obesos e 15 portadores de DMD) com idade entre 6 e 13 anos, mostrou que a porcentagem de gordura desses portadores não foi diferente do grupo de obesos e não obesos normais.

Eiholzer et al. (23) estudaram a evolução do crescimento, de forma retrospectiva, e demonstraram que a pequena estatura é um achado comum na DMD, mesmo no início da vida ou em estágios pré-clínicos da doença. Demonstraram ainda que mesmo crianças nascidas com peso e altura normais apresentam uma curva de crescimento que decai lentamente já no primeiro ano de vida, embora a maturação óssea fosse normal. Decorre que, em função dos achados dos autores, é possível classificar o IMC (índice de massa corporal) dentro da normalidade, caso se desconsidere a idade.

Diante do fato da composição corporal dos portadores de DMD ser alterada, e esta alteração estar associada a diferentes causas, o estudo desta em populações com características próprias pode auxiliar na compreensão da evolução e na condução clínica.

Este estudo teve por objetivo descrever a porcentagem e a massa de gordura avaliada por meio de bioimpedância, bem como correlacionar estes dados com a idade, em uma amostra brasileira de portadores de Distrofia Muscular de Duchenne, moradores da cidade de São Paulo, Brasil.

\section{Método}

\section{Delineamento da pesquisa}

Estudo retrospectivo utilizando banco de dados, cuja coleta fora previamente padronizada por meio de protocolo de avaliação.

\section{Amostra}

Dados relativos a 68 indivíduos com diagnóstico molecular de certeza para Distrofia Muscular de Duchenne, com idade mínima de 5 anos e máxima de 20, não cadeirantes, residentes na cidade de São Paulo, 
em tratamento fisioterapêutico na Associação Brasileira de Distrofia Muscular (ABDIM). Os critérios de exclusão foram: não ter Distrofia Muscular de Duchenne comprovada por DNA, ausência de concordância dos pais ou da criança e não seguir as orientações dadas para o exame de bioimpedância, como, por exemplo, ter tomado café ou comido chocolate. Não houve perdas na amostra.

\section{Procedimento}

Os indivíduos foram submetidos à medição de peso e altura no momento da coleta, utilizando uma balança $\left(\right.$ Fillizolla $\left.^{\circledR}\right)$, e ao teste de análise de composição corporal pela porcentagem de gordura por meio de impedância (Biodinamics $\left.{ }^{\circledR}\right)$, durante o período da manhã, todas no mesmo dia, com a presença e consentimento pós-informado dos pais ou responsável e aceite da criança após demonstração dos procedimentos. Os responsáveis pelos participantes foram orientados verbalmente e por escrito a não fornecerem alimentos ou bebidas como álcool, café, chocolate, chá preto ou diuréticos, e fornecer refeições leves nas 24 horas que precediam o exame. Requisitou-se para não portarem roupas sintéticas, dando preferência às de algodão. A análise de composição corporal foi realizada com o objetivo de mensurar a porcentagem e a massa de gordura corporal.

A técnica de avaliação da composição corporal por meio de impedância elétrica ou bioimpedância não é invasiva, é simples e de rápida aplicação, utilizando equipamento fácil de ser transportado e relativamente barato. Se realizada em condições padronizadas, fornece dados confiáveis e reproduzíveis $(24,25)$, permitindo a quantificação detalhada dos componentes da estrutura corporal $(26,27)$. É considerado um método seguro para avaliação da composição corporal no acompanhamento clínico dos portadores de DMD (28). Todos os dados foram coletados por um único pesquisador devidamente treinado.

\section{Análise de dados}

Para estudo da idade dividiram-se os indivíduos pesquisados em quartis, obtendo-se grupos com idades afins. Realizou-se análise estatística descritiva para as variáveis estudadas.

O percentil de crescimento, o peso, a altura e o IMC (índice de massa corporal) foram comparados com padrões estabelecidos pelo National Center for Health Statistics (NCHS) e CDC Growth Charts: United States (2000), elaborados para indivíduos com idade entre 2 e 20 anos. A massa de gordura encontrada foi comparada com dados publicados por McDonald (22) em um estudo de avaliação da composição corporal com 46 indivíduos participantes, sendo 12 não obesos, 19 obesos e 15 portadores de DMD com idade entre 6 e 13 anos, e a porcentagem de gordura foi comparada com os valores fornecidos por Deurenberg (28) a partir de estudo de 378 meninos e meninas normais, com idade entre 7 e 20 anos.

\section{Resultados e discussão}

Após a divisão dos indivíduos em quartis, encontrando quatro grupos com idades afins, foi realizada análise estatística descritiva para as variáveis estudadas (Tabela1). Os participantes do Grupo 1 (G1), com idades entre 5 e 8 anos, apresentaram altura de $122 \pm 7,6 \mathrm{~cm}$, o que é considerado eutrofia (normal) segundo Krause (25) e a tabela do NCHS. Para esse grupo, verificou-se que a DMD não interferiu no desenvolvimento do crescimento, expresso pela altura. O peso dessas crianças foi 22,71 $\pm 5,78 \mathrm{~kg}$ e o índice de massa corpórea (IMC) $14 \pm 0,02 \mathrm{~kg} / \mathrm{m}^{2}$, com percentil $46^{\circ}$, considerados normais conforme a literatura. A porcentagem de gordura desse grupo foi de $41,1 \pm 20,5 \%$ encontrando-se elevada quando comparada com os valores descritos por Deurenberg (28). A massa de gordura foi de 8,46 \pm 6,21 kg, compatível com o encontrado no estudo de McDonald (22), cujos valores apresentados foram de 10,5 $\pm 2,1 \mathrm{~kg}$. 
Tabela 1 - Comparação dos valores obtidos com traqueias de diferentes diâmetros

\begin{tabular}{|c|c|c|c|c|c|}
\hline & \multicolumn{4}{|c|}{ Desvio-padrão } & \multirow[t]{2}{*}{ Média } \\
\hline & $\begin{array}{c}\text { G1 } \\
5 \text { a } 8 \text { anos }\end{array}$ & $\begin{array}{c}\text { G2 } \\
9 \text { a } 11 \text { anos }\end{array}$ & $\begin{array}{c}\text { G3 } \\
12 \text { a } 13 \text { anos }\end{array}$ & $\begin{array}{c}\text { G4 } \\
14 \text { a } 20 \text { anos }\end{array}$ & \\
\hline Altura (cm) & $122 \pm 7,6$ & $130,5 \pm 9,3$ & $141,3 \pm 13,5$ & $157 \pm 10,5$ & $139,2 \pm 17$ \\
\hline Peso $(k g)$ & $22,71 \pm 5,78$ & $37,81 \pm 11,84$ & $36,83 \pm 10,59$ & $57,29 \pm 15,59$ & $40,83 \pm 17,44$ \\
\hline $\operatorname{IMC}\left(\mathrm{P} / \mathrm{H}^{2}-\mathrm{kg} / \mathrm{m}^{2}\right)$ & $14 \pm 0,02$ & $21 \pm 0,2$ & $21 \pm 0,11$ & $24 \pm 0,7$ & $21 \pm 0,8$ \\
\hline Porcentagem de gordura & $41,1 \pm 20,5$ & $52,4 \pm 11,4$ & $48,4 \pm 17,9$ & $49,7 \pm 12,3$ & $48,5 \pm 15,3$ \\
\hline Massa (kg) & $8,46 \pm 6,21$ & $19,52 \pm 7,35$ & $17,95 \pm 9,47$ & $28,48 \pm 10,11$ & $19,87 \pm 11,04$ \\
\hline
\end{tabular}

No Grupo 2 (G2), com participantes com idades entre 9 e 11 anos, a altura foi de 130,5 \pm 9,3 cm, compatível com os valores da normalidade (29). Apresentaram peso de 37,81 $\pm 11,84 \mathrm{~kg}$, considerado acima da normalidade. Essas crianças apresentaram IMC de $21 \pm 0,2 \mathrm{~kg} / \mathrm{m}^{2}$ e percentil de $95^{\circ}$, estando classificadas como obesas segundo a tabela NCHS. A porcentagem de gordura encontrada foi de $52,4 \pm 11,4 \%$ e a massa de gordura, de 19,52 $\pm 7,35 \mathrm{~kg}$.

Os participantes do Grupo 3 (G3), com idades de 12 e 13 anos, apresentaram altura de 141,3 \pm 13,5 $\mathrm{cm}$, verificando-se que houve um déficit no crescimento. A porcentagem de gordura foi de 48,4 $\pm 17,9 \%$.

Os participantes do Grupo 4 (G4), com idades entre 14 e 20 anos, apresentaram altura de $157 \pm$ $10,5 \mathrm{~cm}$, com déficit no crescimento concordando com Zatz (30). O peso foi de 57,29 $\pm 15,59 \mathrm{~kg}$ e IMC 24 $\pm 0,7 \mathrm{~kg} / \mathrm{m}^{2} \mathrm{com}$ percentil $70^{\circ}$ considerados normais conforme NCHS. Encontrou-se aumento na porcentagem de gordura, 49,7 $\pm 12,3 \%$, verificando-se que a porcentagem de gordura nos indivíduos com DMD é aumentada. A massa de gordura desse grupo foi de 28,48 $\pm 10,11 \mathrm{~kg}$.

Na população estudada, podemos afirmar que nas crianças de 5 a 8 anos de idade (grupo 1) a DMD não interferiu no crescimento. Isso contradiz os resultados de Eiholzer (23), que mostraram que a pequena estatura é um achado comum na DMD e também não interferiu no peso dessas crianças. Elas, no entanto, já iniciavam o acúmulo de gordura característico da doença, dados compatíveis com os achados de Cole (31), que definiu o padrão de sobrepeso e obesidade em um estudo com 97.876 homens e 94.851 mulheres com idade de zero a 25 anos.

O que nos chamou a atenção nos indivíduos com idades entre 9 e 11 anos (G2) foi o aumento na porcentagem de gordura e na massa com quadro de obesidade, provavelmente desencadeada pela diminuição da atividade física.

Os indivíduos com idades entre 12 e 13 anos (G3) apresentaram diminuição no crescimento e peso adequado para faixa etária (o que caracterizava meninos pequenos). A porcentagem e a massa de gordura também se encontraram acima dos valores normais.

Os participantes com idade entre 14 e 20 anos (G4) também apresentaram uma diminuição no crescimento, peso e IMC compatíveis com a idade e aumento na porcentagem e na massa de gordura.

\section{Conclusão}

Conforme a idade e o grau de sedentarismo imposto pela doença, os participantes passaram a acumular gordura corporal. Como esse aumento não se refletiu no peso, acredita-se que ocorreu perda de massa magra, especialmente dos tecidos do sistema músculo-esquelético. 
Sugerimos estudos relacionando idade, composição corporal, dados antropométricos e características nutricionais, que poderão esclarecer melhor a influência da doença e da alimentação no ganho de porcentagem e massa de gordura.

\section{Referências}

1. Emery AEH. Duchenne muscular dystrophy. 2nd ed. Oxford: Oxford University Press; 1993.

2. Fonseca JG, Machado MJF, Ferraz CLMS. Distrofia Muscular de Duchenne: complicações respiratórias e seu tratamento. Rev Ciênc Méd Campinas. 2007;16(2):109-11.

3. Centers for Disease Control and Prevention (CDC). Prevalence of Duchenne/Becker Muscular Dystrophy among males aged 5-24 years - Four States, 2007. MMWR Morb Mortal Wkly Rep. 2009;58(40):1119-22.

4. Pena FF, Rosolém FC, Alpino AMS. Contribuição da Fisioterapia para o bem-estar e a participação de dois alunos com Distrofia Muscular de Duchenne no ensino regular. Rev Bras Ed Esp. 2008;14(3):447-15.

5. Tonelli D, Pinho I, Sacco P de CN, Vianna EP, Vasconcellos JC de, Souza RV de, et al. Anestesia em Distrofia Muscular de Duchenne; Relato de Caso. Rev Bras de Anest. 2003;53(3):392-3.

6. Willing TN, Curlier L, Legratirl AL, Riviere H, Navarro J. Nutritional assessment in Duchenne Muscular Dystrophy. Dev Med Child Neurol. 1993;35(12):1074-82.

7. Rideau YM. Outlines of muscular dystrophy. France Diagnosis. 1978;13(9):177-86.

8. Griffiths RD, Edwards DH. A new chart for weight control in Duchenne Muscular Dystrophy. Arch Dis Child. 1988:63(10):1256-2.

9. Moreira ASS, Araújo APQC. Não reconhecimento dos sintomas iniciais na atenção primária e a demora no diagnóstico da Distrofia Muscular de Duchenne. Rev Bras Neurol. 2009:45(3):39-4.

10. Silva CMC. Pereira AC, Araújo APQC. Distrofia Muscular de Duchenne: fatores da história associados ao diagnóstico precoce. Rev Bras Neurol. 2006;42(4):35-4.

11. Araújo AP de QC, Deco MC de, Klôh B de S, Costa MR da, Góis FV de, Guimarães AFCM. Diagnosis delay of Duchenne Muscular Dystrophy. Rev Bras de Saúde Materno Infantil. 2004;4(2):179-4.

12. DavidsonZE, Truby H. A reviem of nutrition in Duchenne Muscular Dystrophy.J Hum Nutr Diet. 2009;22(5):383-10.

13. Skalsky AJ, Han JJ, Abresch RT, Shin CS, Mcdonald CM. Assessment of Regional body composition with dualenergy X-Ray Absorptiometry in Duchenne Muscular Dystrophy: correlation of regional Lean Mass and quantitative strength. Muscle Nerve. 2009:39(5):647-4.

14. Hsieh TJ, Jaw TS, Chuang HY, Jong YJ, Liu GC, Li CW, Muscle metabolism in Duchenne Muscular Dystrophy assessed by in vivo proton magnetic resonance spectroscopy. J Comput Assist Tomogr. 2009;33(1):150-4.

15. Hogan SE. Body composition and resting energy expenditure of individuals with Duchenne and Becker Muscular Dystrophy. Can J Diet Pract Res. 2008; 69(4):208-12.

16. Radley HG, De Luca A, Lynch GS, Grounds MD. Duchenne Muscular Dystrophy: focus on pharmaceutical and nutritional interventions. Int J Biochem Cell Biol. 2007;39(3):469-8.

17. Zanardi MC, Tagliabue A, Orcesi S, Berardinelli A, Uggetti C, Pichiecchio A. Body composition and energy expendeture in Duchenne Muscular Dystrophy. Original communication. Acta Diabetol. 2003;40(Suppl 1):S290-2.

18. Hankard R, Gottrand F, Turck D, Carpentier A, Romon M, Farriaux JP. Resting energy expenditure and energy substrate utilization in children with Duchenne Muscular Dystrophy. Pediatr Res. 1996;40(1):29-33. 
19. Motlagh B, MacDonald JR, Tarnopolsky MA. Nutritional inadequacy in adults with Muscular Dystrophy. Muscle Nerve. 2005;31(6):713-8.

20. Satomura S, Yokota I, Tatara K, Naito E, Ito M, Kuroda Y. Paradoxal weight loss with extra energy expenditure at brown adipose tissue in adolescent Duchenne Muscular Dystrophy. Metabolism. 2001;50(10):1181-5.

21. Berlit P, Lutzman D, Kuhn C, Leweling H. Bioeletric impedance analysis: method for diagnosis and follow-up of muscular distrophies. Proceedings VII International Congress on Neuromuscular Diseases, 16-22 September, 1990; Munich. Springer Netherlands; 1990.

22. McDonald CM, Carter GT, Abresch RT, Widman L, Styne DM, Warden N, et al. Body Composition and water compartment measurements in boys with Duchenne Muscular Dystrophy. Am J Phys Med Rehabil. 2005;84(7): 483-91.

23. Eiholzer U, Boltshauser E, Frey D, Molinari L, Zachmann M. Short stature: a common feature in Duchenne Muscular Dystrophy. Eur J Pediatric. 1988;147(6):602-5.

24. Segal KR, Loan MV, Fitz Gerald PI, Hodgdon JA, Itallie TBV. Lean body mass estimation by bioelectrical impedance analysis: a four-site-validation study. Am J Clin Nutr. 1988;47(1):7-14.

25. Lukaski HC, Bolonchuk WW, Hall CB, Siders WA. Validation of tetrapolar bioelectrical impedance method to assess human body composition. J Appl Physiol. 1986;60(4):1327-5.

26. Mcardle, WD, Katch, FI, Katch, VL. Exercise physiology, energy nutrition, and human performance. 3rd ed. Philadelphia: Lea \&Febiger; 1991.

27. Basttini, D, Ballardini D, Rosati R, Ballestrazzi A, Campo G, Colombo C, et al. Body composition analysis in Duchenne Muscular Dystrophy (DMD). Bologna, Italy: Institute of Physiology; 1990.

28. Deurenberg P, Pieters JJL and Hautvast JGAJ. The assessment of the body fat percentage by skinfold thickness measurements in childhood and young adolescence. Netherlands: University, Netherlands; 1990.

29. Mahan K, Escott S. Krause alimentos, nutrição e dietoterapia. 10a. ed. São Paulo: Roca; 2002.

30. Zatz, M. Relação da estatura e peso com o desempenho muscular e os níveis enzimáticos na Distrofia Muscular de Duchenne. São Paulo: Edusp; 1987.

31. Cole TJ, Bellizzi MC, Flegal KM, Dietz WH. Establishing a standard definition for child overweight and obesity worldwide international survey. BMJ. 2000;320(7244):1240-3.

Recebido: 30/06/2008

Received: 06/30/2008

Aprovado: 04/11/2009

Approved: 11/04/2009

Revisado: $10 / 05 / 2010$

Reviewed: 05/10/2010 